EPJ Web of Conferences 31, 00031 (2012)

DOI: $10.1051 /$ epjconf/20123100031

(C) Owned by the authors, published by EDP Sciences - SIF, 2012

\title{
R3B time projection chamber
}

\author{
P. LEGOU
}

CEA Saclay, France

\begin{abstract}
Within the international FAIR project, the R3B collaboration (Reaction studies with Radioactive Relativistic beams) will be in charge of the physics program with secondary beams of energy between 200 and $1500 \mathrm{MeV}$ per nucleon. Central to the R3B set-up will be a largeaperture superconducting magnet under construction at CEA-Saclay. An international collaboration has been formed to work on the design of a large time-projection chamber (TPC) to be installed behind this magnet to cover the full phase-space of the charged fragments produced in the target. Within this collaboration, tests of detector prototypes were performed with the heavy-ions beams at GSI Darmstadt. These prototypes were equipped with a gaseous Micromegas detector. Two amplification technologies were tested, either conventional or resistive Micromegas and two construction concepts, bulk-Micromegas or micro-meshes screwed on the PCB. We will present the results of the last beam and the $1 \mathrm{~m}$ TPC station which will be dedicated to make tests using the real drift distance and compare the performances of micromegas and GEM.
\end{abstract}

This is an Open Access article distributed under the terms of the Creative Commons Attribution License 2.0, which permits unrestricted use, distribution, and reproduction in any medium, provided the original work is properly cited. 